\title{
Spectral reflection and dot surface prediction models for color halftone prints
}

\author{
Roger D. Hersch \\ Ecole Polytechnique Fédérale de Lausanne (EPFL) \\ Switzerland \\ Patrick Emmel \\ Clariant International \\ Muttenz \\ Switzerland \\ Fabien Collaud \\ Frédéríque Crété \\ Ecole Polytechnique Fédérale de Lausanne (EPFL)
}

Switzerland

\begin{abstract}
The proposed new spectral reflection model enhances the classical Clapper-Yule model by taking into account the fact that proportionally more incident light through a given colorant surface is reflected back onto the same colorant surface than onto other colorant surfaces. It comprises a weighted mean between a component specifying the part of the incident light that exits through the same colorant as the colorant from which it enters (Saunderson corrected Neugebauer component) and a component specifying the part of the incident light whose emerging light components exit from all colorants (Clapper-Yule component). We also propose models for taking into account ink spreading, a phenomenon that occurs when printing an ink halftone in superposition with one or several solid inks. The ink-spreading model incorporates nominal-to-effective surface coverage functions for each of the different ink superposition conditions. A system of equations yields the effective ink surface coverages of a color halftone as a weighted mean of the ink surface coverages specific to the different superposition conditions. The new spectral reflection prediction model combined with the ink-spreading model yields excellent spectral reflection predictions for clustered-dot color halftones printed on an offset press or on thermal transfer printers. () 2005 SPIE and IS\&T. [DOI: 10.1117/1.1989987]
\end{abstract}

\section{Introduction}

For more than 50 years, attempts have been made to build models predicting the color of printed halftone images. To offer accurate predictions, the models must take into account, at least to some extent, the phenomena determining the interactions of inks and paper and of light and halftone prints.

Many different phenomena influence the reflection spectrum of a color halftone patch printed on a diffusely reflecting substrate (e.g., paper). These phenomena comprise the surface (Fresnel) reflection at the interface between the air and the paper, light scattering and reflection within the sub-

Paper 04159R received Oct. 1, 2004; revised manuscript received Jan. 14, 2005; accepted for publication Jan. 24, 2005; published online Aug. 11, 2005 .

1017-9909/2005/14(3)/033001/12/\$22.00 @ 2005 SPIE and IS\&T. strate (i.e., paper bulk), and the internal (Fresnel) reflections at the interface between the paper and the air. The lateral scattering of light within the paper substrate and the internal reflections at the interface between the paper and the air are responsible for the portion of light that enters the print through an inked dot and exits the print through the paper white and vice versa. This effect yields the optical dot gain also known as the "Yule-Nielsen" effect. In addition, due to the printing process, the deposited ink surface coverage is generally larger than the nominal coverage, yielding a "physical" dot gain (sometimes also called "mechanical" dot gain). Effective ink surface coverages depend on the inks, on the paper, and also on the specific superpositions of the different inks.

Currently, according to the literature, ${ }^{1,2}$ among the existing spectral reflection prediction models, only the wellknown Yule-Nielsen modified Neugebauer model ${ }^{3-6}$ seems to be used in practice. Most other spectral prediction models (see Sec. 2) enable us to explore various effects, but are either too complex or not accurate and comprehensive enough to be usable in practice.

The model we propose is an enhancement of the classical Clapper-Yule model that models optical dot gain of halftone prints by taking into account lateral scattering within the paper bulk and multiple internal reflections. Our model also takes into account the different physical dot surface coverages that occur under different ink superposition conditions.

We developed our prediction model by adding successive enhancements to the basic Clapper-Yule model. The benefits of each of these enhancements is verified by comparing measured halftone patch reflection spectra and predicted spectra, for 729 patches covering the full printable gamut, produced by generating all combinations of inks at nominal coverages of $0,13,25,38,50,63,75,88$, and $100 \%$. We quantify the visual quality of color halftone 
patch predictions by converting measured and predicted spectra first to Commission International de l'Eclairage (CIE) $X Y Z$ and then to CIELAB (Ref. 7, pp. 8-12). The Euclidian distance $\Delta E_{a b}^{*}$ in CIELAB (1976) space gives a measure of the visually perceived distance between measured and predicted spectra. To derive successive model improvements, we proceed as follows. When predictions and measurements disagree, we observe the corresponding predicted and measured reflection spectra, try to give an explanation of the underlying phenomenon (e.g., ink spreading), and propose an appropriate modeling step. The measurements are carried out with a photospectrometer having a $45 \mathrm{deg} / 0 \mathrm{deg}$ geometry, i.e., with a D65 light source illuminating the printed sample at an angle of $45 \mathrm{deg}$ and a sensor capturing the reflected spectrum at 0 deg (normal to the printed sample).

\section{Existing Approaches to Spectral Color Prediction}

In early prediction models of color halftone prints, the term "dot gain" encompasses both the physical dot gain (the enlargement of the printed dot) and the optical dot gain due to the lateral propagation of light. Lateral propagation of light occurs due to scattering within the paper bulk and to internal reflections at the print-air interface. The Neugebauer model $^{8}$ predicts the CIE $X Y Z$ tristimulus values of a color halftone patch as the sum of the tristimulus values of their individual colorants weighted by their fractional area coverages $a_{i}$. By considering instead of the tristimulus values of colorants their respective reflection spectra $R_{i}$, one obtains the spectral Neugebauer equations. They predict the reflection spectrum of a printed color halftone patch as a function of the reflection spectra of its individual colorants

$R(\lambda)=\sum_{i} a_{i} R_{i}(\lambda)$

In the case of independently printed cyan, magenta, and yellow inks of respective coverages $c, m$, and $y$, the fractional area coverages of the individual colorants are closely approximated by the Demichel Eqs. (2), which give the respective surface coverages of the colorants as a function of the surface coverages of the individual inks. ${ }^{9}$ The respective fractional areas of the colorants white, cyan, magenta, yellow, red (superposition of magenta and yellow), green (superposition of yellow and cyan), blue (superposition of magenta and cyan), and black (superposition of cyan, magenta and yellow) are, respectively,

$$
\begin{aligned}
\text { white: } & a_{w}=(1-c)(1-m)(1-y), \\
\text { cyan: } & a_{c}=c(1-m)(1-y), \\
\text { magenta: } & a_{m}=(1-c) m(1-y), \\
\text { yellow: } & a_{y}=(1-c)(1-m) y, \\
\text { red: } & a_{r}=(1-c) m y, \\
\text { green: } & a_{g}=c(1-m) y, \\
\text { blue: } & a_{b}=c m(1-y), \\
\text { black: } & a_{k}=c m y .
\end{aligned}
$$

The Neugebauer model is a generalization of the MurrayDavis model, ${ }^{10}$ whose colorants are formed by only one ink and the paper white.
Since the Neugebauer model takes explicitly into account neither the lateral propagation of light within the paper bulk nor the internal reflections (Fresnel reflections) at the paper-air interface, its predictions are not accurate. ${ }^{11}$ Yule and Nielsen ${ }^{3}$ modeled the nonlinear relationship between the reflection spectra of paper and solid ink and the reflection spectra of single ink halftones by a power function, whose exponent $n$ is fitted according to a limited set of measured halftone patch reflection spectra. Viggiano ${ }^{4}$ applied the Yule-Nielsen relationship to the spectral Neugebauer equations, yielding the Yule-Nielsen modified Spectral Neugebauer model:

$R(\lambda)=\left(\sum_{i} a_{i} R_{i}(\lambda)^{1 / n}\right)^{n}$

This Yule-Nielsen modified spectral Neugebauer model has been used by several researchers for the characterization of various printers. ${ }^{2,4-6}$ This model seems to play a significant role for building color management systems.

Ruckdeschel and Hauser ${ }^{12}$ analyzed the Yule-Nielson model by modeling the lateral propagation of light within the paper bulk by a point spread function $H(x, y)$. They proposed the following 2-D convolution integral for the reflection spectrum $R$ at position $(x, y)$ :

$R(x, y)=T(x, y) R_{p} \int_{-\infty}^{\infty} \int_{-\infty}^{\infty} T\left(x^{\prime}, y^{\prime}\right) H\left(x-x^{\prime}, y-y^{\prime}\right) d x^{\prime} d y^{\prime}$

Light enters at all positions $\left(x^{\prime}, y^{\prime}\right)$, located in the neighborhood of $(x, y)$, is attenuated by the ink transmittance $T\left(x^{\prime}, y^{\prime}\right)$, enters the paper bulk, propagates laterally with a fraction $H\left(x-x^{\prime}, y-y^{\prime}\right)$ reaching position $(x, y)$, is attenuated according to the paper reflectance $R_{p}$, exits the paper bulk, is attenuated by the ink layer transmittance $T(x, y)$ and emerges at position $(x, y)$. The integral simply sums up the contributions of all light components incident at positions $\left(x^{\prime}, y^{\prime}\right)$, attenuated by $H\left(x-x^{\prime}, y-y^{\prime}\right)$, which exit at position $(x, y)$. Much research was carried out in building models by assuming various mathematical formulations of the point spread function $H(x, y)$. Both Gaussian line spread functions ${ }^{12,13}$ and exponential point spread functions were proposed. ${ }^{14-16}$ Since the point spread function can also be viewed as a probability density, probability models were proposed to describe the lateral scattering of light within the paper bulk. ${ }^{17-20}$

The models described by Eq. (4) assume that light traverses the ink layer, is laterally scattered and reflected by the paper, traverses a second time the ink layer (at a different position due to lateral scattering), and exits from the printed paper. However, as is known from optics, Fresnel reflections occur at a planar interface between two media of different indices of refraction. Paper is formed by a network of layered cellulose fibers plus filler pigments and is often coated with a coating whose index of refraction is generally assumed to be 1.53 .

In this context, we consider the Clapper-Yule model, ${ }^{21}$ which was developed to predict the reflectance of photographic prints. The Clapper-Yule model has the advantage of modeling the specular reflections at the air-paper inter- 


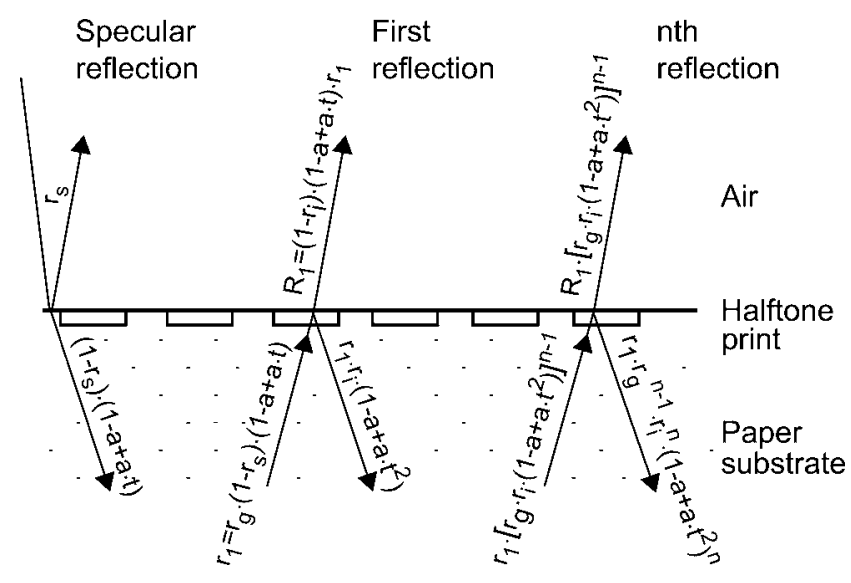

Fig. 1 Attenuation of light by multiple reflections on a halftone printed patch. Specular reflection: $r_{s}$. First reflection: $\left(1-r_{s}\right) r_{g}\left(1-r_{i}\right)$ $\times(1-a+a t)^{2}$. Second reflection: $\left(1-r_{s}\right) r_{g}\left(1-r_{i}\right)(1-a+a t)^{2} r_{i} r_{g}(1-a$ $\left.+a t^{2}\right)$. nth reflection: $\left(1-r_{s}\right) r_{g}\left(1-r_{i}\right)(1-a+a t)^{2}\left(r_{i} r_{g}\left(\left(1-a+a t^{2}\right)\right)^{n-1}\right.$.

face and the multiple internal reflections between the paper bulk and the paper-air interface (Fresnel reflectivity). It assumes that lateral light propagation due to light scattering within the paper bulk is large compared with the period of the halftones. Therefore, the probability of light exiting from a given colorant is set equal to the colorant's fractional surface coverage.

Rogers $^{22}$ generalizes the Clapper-Yule model by modeling lateral scattering within the paper as a point spread function and by deducing the probabilities that light entering through a colorant $n$ emerges from the coated paper through a colorant $m$, possibly traversing, due to multiple reflections, further intermediate colorants. Emmel and Hersch's unified model ${ }^{23}$ also takes into account multiple internal reflections and modelizes lateral scattering probabilities by an exponential function with a circular symmetry and a strong radial decay.

Within the framework of their work on the reproduction of color images by custom inks, Stollnitz et al. ${ }^{24}$ predict the reflection spectra of solid colorants by using Kubelka's layering model ${ }^{25}$ to combine the paper layer and the ink layers and by applying Saunderson's correction ${ }^{26}$ to take into account multiple reflections at the interface between the paper coating and the air. They predict halftone colors by combining the resulting solid colorant colors according to the Neugebauer equations, extended so as to account for dot gain and trapping.

\section{Basic Clapper-Yule Spectral Color Prediction Model}

Among the classical color prediction models, ${ }^{11}$ only the Clapper-Yule model ${ }^{21}$ takes simultaneously into account halftone patterns and multiple internal reflections occurring at the interface between the coated paper and the air.

To introduce the Clapper-Yule model, we consider a single halftone ink layer with a fractional surface coverage $a$ printed on a coated paper substrate (Fig. 1). Incident light has the probability $a$ of reaching the paper substrate by passing through ink of transmittance $t(\lambda)$ and a probability $(1-a)$ of reaching the substrate without traversing the ink layer. Since $r_{s}$ is the specular reflection at the air-paper interface, only portion $\left(1-r_{s}\right)$ actually enters the coated paper. The light reaching the paper substrate is reduced by a factor $\left(1-r_{s}\right)(1-a+a t)$. It is diffusely reflected by the paper substrate according to the paper substrate reflectance $r_{g}(\lambda)$. Traveling upward, it traverses the coated paper with a portion $a$ traversing the ink and a portion $1-a$ traversing an area free of ink. It is reflected at the coated paper-air interface according to a reflection factor $r_{i}$ (Fresnel reflectivity integrated over all incident angles). A part $\left(1-r_{i}\right)$ of the light exits. At the first exit, the spectral attenuation of the incident light is therefore $\left(1-r_{s}\right) r_{g}\left(1-r_{i}\right)(1-a+a t)^{2}$. The part reflected at the coated paper-air interface travels downward, is diffusely reflected by the paper, and travels upward again. At the second exit, the spectral attenuation is $\left(1-r_{s}\right) r_{g}\left(1-r_{i}\right)(1-a+a t)^{2} r_{i} r_{g}\left(1-a+a t^{2}\right)$.

With $K$ giving the fraction of specular reflected light reaching the photospectrometer (when the specular component is discarded by the photospectrometer, we set $K=0$ ), and by considering the light emerging after $0,1,2, \ldots, n$ -1 internal reflections (Fig. 1), we obtain the reflection spectrum

$$
\begin{aligned}
R(\lambda)= & K r_{s}+\left[\left(1-r_{s}\right)\left(1-r_{i}\right) r_{g}(1-a+a t)^{2}\right] \\
& \times\left[1+\left(r_{i} r_{g}\left(1-a+a t^{2}\right)\right]+\left[r_{i} r_{g}\left(1-a+a t^{2}\right)\right]^{2}\right. \\
& +\cdots+\left[r_{i} r_{g}\left(a+a t^{2}\right)\right]^{n-1} .
\end{aligned}
$$

For an infinite number of emergences (geometric series), we obtain the Clapper-Yule expression

$R(\lambda)=K r_{s}+\frac{\left(1-r_{s}\right) r_{g}\left(1-r_{i}\right)(1-a+a t)^{2}}{1-r_{g} r_{i}\left(1-a+a t^{2}\right)}$

The light components that have been summed in Eq. (5) are fractions of the incident irradiance. Therefore, Eq. (5) expresses a reflectance, i.e., the ratio between exiting irradiance and incident irradiance. Ideally, incident and exiting irradiances should be measured with a spectrophotometer having an integrated sphere geometry. However, as shown in Appendix B, when the specular component is discarded $(K=0)$, for the index of refraction $n=1.53$ of coated paper, the exact expression for the spectral reflectance factor predicted according to a $45 \mathrm{deg} / 0 \mathrm{deg}$ measuring geometry ${ }^{27}$ is numerically very similar to Eq. (5). One can therefore, in the context of color reproduction, carry out all measurements with a photospectrometer having a $45 \mathrm{deg} / 0 \mathrm{deg}$ geometry, i.e., an instrument where light illuminates the printed sample at an orientation of $45 \mathrm{deg}$ and where reflected light (radiance) is captured at an orientation normal to the sample.

In the case of paper printed with three inks such as cyan, magenta, and yellow, the surface coverages $a_{j}$ of the resulting eight basic colorants, i.e., white*, cyan, magenta, yellow, red, green, blue, and black are obtained according to the Demichel Eqs. (2). By inserting the relative amounts of colorants $a_{j}$ and their transmittances $t_{j}$ in Eq. (5), we obtain for the predicted reflectance of a color patch printed with combinations of cyan, magenta, and yellow inks

*The internal transmittance $t_{w}$ of white (no ink) is 1 at each wavelength. 


$$
R(\lambda)=K r_{s}+\frac{\left(1-r_{s}\right) r_{g}\left(1-r_{i}\right)\left(\sum_{j=1}^{8} a_{j} t_{j}\right)^{2}}{1-r_{g} r_{i} \sum_{j=1}^{8} a_{j} t_{j}^{2}} .
$$

Both the specular reflection $r_{s}$ and the internal reflection $r_{i}$ at the paper-air interface depend on the refraction indices of the air $\left(n_{1}=1\right)$ and of the coated paper $\left(n_{2}=1.53\right)$, independently of whether the considered surface is white or printed (the ink is located within the coated paper surface). According to the Fresnel equations, ${ }^{28}$ for collimated light at an incident angle of $45 \mathrm{deg}$, the specular reflection factor is $r_{s}=0.054$. With light diffusely reflected by the paper (Lambert radiator), by summing the contributions at all incident angles, ${ }^{27}$ we obtain the internal reflection factor $r_{i}$. For coated paper, $r_{i}=0.614$. Values of $r_{i}$ for different indices of refraction are tabulated by Judd ${ }^{29}$ and by Emmel. ${ }^{30}$

To put the model into practice, we deduce from Eq. (5) the internal reflectance spectrum $r_{g}$ of a blank paper by setting the ink coverage $a=0$. Here, $R_{w}$ is the measured blank paper reflectance.

$r_{g}=\frac{R_{w}-K r_{s}}{1+(1-K) r_{i} r_{s}+r_{i} R_{w}-r_{s}-r_{i}}$.

We then extract the transmittance of the individual solid inks and solid ink superpositionsa $t_{w}, t_{c}, t_{m}, t_{y}, t_{r}, t_{g}, t_{b}$, and $t_{k}$ by inserting in Eq. (5) as $R(\lambda)$ the measured solid (100\%) ink (respectively solid ink superpositions) reflectance $R_{i}$ and by setting the ink coverage $a=1$ :

$t_{i}=\left[\frac{R_{i}-K r_{s}}{r_{g} r_{i}\left(R_{i}-K r_{s}\right)+r_{g}\left(1-r_{i}\right)\left(1-r_{s}\right)}\right]^{1 / 2}$.

We must also take a possible physical dot gain into account. For each ink, we fit ${ }^{\dagger}$ according to Clapper-Yule [Eq. (5)] the unknown physical surface coverages of the measured single ink patches at nominal surface coverages of for example $10,20, \ldots, 90 \%$ by minimizing the sum of square differences between measured and predicted spectra components (similar to the dot area optimization proposed by Balasubramanian $^{2}$ ). For the basic Clapper-Yule model, fitted single ink surface coverages are lower than the nominal surface coverages, i.e., we obtain a negative dot gain [Fig. 2(a)]. This is due to the fact that spectra predicted by the Clapper-Yule model are darker than the corresponding measured spectra. The fitted negative dot gain tends to bring both spectra to the same levels, i.e., the predicted and measured spectra intersect each other [Fig. 2(b)].

To set a base line for improvements, we test the accuracy of the basic Clapper-Yule model taking into account single ink dot gain (i.e., single ink physical dot surface optimization, see Ref. 2, Section 4) by printing at the considered screen frequencies a set of 729 patches. Screen frequencies are expressed in lines per inch (lpi). These patches are produced by generating all combinations of cyan, magenta, and yellow ink superpositions at nominal coverages $0,13,25,38,50,63,75,88$, and 100\%. Measured and predicted spectra are converted to CIELAB (1976) values

\footnotetext{
Fitting of a surface coverage is carried out with MATLAB's fminsearch function, which uses the downhill simplex search method to find the surface coverage yielding the minimum of the function expressing the sum of square differences between measured and predicted reflection spectra components.
}

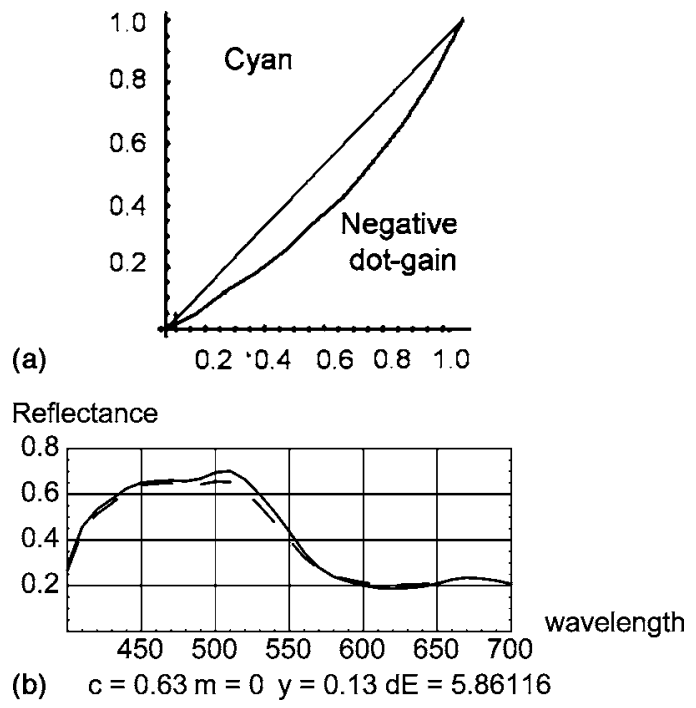

Fig. 2 (a) Negative dot gain induced by the too dark Clapper-Yule predictions and (b) corresponding measured (continuous) and predicted (dashed) reflection spectra.

and the resulting prediction error $\Delta E_{a b}^{*}$ is computed. With the Clapper-Yule model, for offset prints at a screen frequency of $150 \mathrm{lpi}$, a mean error of $\Delta E_{a b}^{*}=3.95$ is obtained, the maximal error is 10.98 and 352 values have a $\Delta E_{a b}^{*}$ greater than 4 . At a screen frequency of $75 \mathrm{lpi}$, a mean error of $\Delta E_{a b}^{*}=4.49$ is obtained, the maximal error is 9.19 and 438 values have a $\Delta E_{a b}^{*}$ greater than 4 (see Appendix A, Table 1).

\section{New Spectral Color Prediction Model}

Spectra predicted by the Clapper-Yule model (without the negative dot gain compensation) are too dark, because according to the modulation transfer function of paper, ${ }^{31}$ light does not travel significantly more than $0.13 \mathrm{~mm}$ (95th percentile according to the Gaussian line spread function model of Ruckdeschel and Hauser ${ }^{12}$ ) within coated paper. With screen frequencies between 20 to 60 lines per $\mathrm{cm}$ (50 to $150 \mathrm{lpi}$ ), the probability that light having entered at a position having a certain ink color exits from a position of the same color is higher than the coverage of that ink color. Therefore, the basic assumption of the Clapper-Yule model, i.e., the probability of light exiting from a specific colorant being equal to that colorant coverage, is not fulfilled.

To enhance the basic Clapper-Yule model, we assume that a certain part $b$ of the incident light through a given colorant is reflected back and exits from the same colorant. The part $(1-b)$ of the incoming light behaves in the same way as in the basic Clapper-Yule model described in the preceding. We also make the simplifying assumption that the part $b$ of the incident light that is reflected onto the same colorant also exits from the same colorant after one or several reflections at the paper-air interface.

Again taking multiple reflections into account, the attenuation of the part of the incoming light exiting from the same ink color (either no ink or ink with coverage $a$ ) at the first exit is 
Table 1 Prediction accuracy $\left(\Delta E_{a b}^{\star}\right)$ for offset cyan, magenta, and yellow halftone prints, printed on matte coated offset paper.

\begin{tabular}{|c|c|c|c|c|c|c|}
\hline $\begin{array}{l}\text { Offset printing } \\
729 \text { test samples }\end{array}$ & $\begin{array}{c}\operatorname{Max} \\
\Delta E\end{array}$ & $\begin{array}{l}\text { Mean } \\
\Delta E\end{array}$ & $\begin{array}{c}\text { Number of } \\
\text { Samples } \\
\Delta E>4\end{array}$ & $\begin{array}{c}\operatorname{Max} \\
\Delta E\end{array}$ & $\begin{array}{l}\text { Mean } \\
\Delta E\end{array}$ & $\begin{array}{c}\text { Number of } \\
\text { Samples } \\
\Delta E>4\end{array}$ \\
\hline & \multicolumn{3}{|c|}{75 Ipi, b=0.6 } & \multicolumn{3}{|c|}{150 Ipi, b=0.1 } \\
\hline Clapper-Yule with single-ink dot gain only & 9.19 & 4.49 & 438 & 10.98 & 3.95 & 352 \\
\hline $\begin{array}{l}\text { Clapper-Yule, dot gain and ink spreading } \\
\text { according to print order }\end{array}$ & 7.87 & 2.76 & 162 & 4.65 & 2.06 & 20 \\
\hline $\begin{array}{l}\text { Clapper-Yule, dot gain and ink spreading } \\
\text { for all superposition conditions }\end{array}$ & 8.05 & 2.44 & 129 & 4.23 & 1.70 & 6 \\
\hline $\begin{array}{l}\text { New spectral prediction model, with } \\
\text { single-ink dot gain only }\end{array}$ & 4.59 & 2.13 & 7 & 10.15 & 3.55 & 292 \\
\hline $\begin{array}{l}\text { New spectral prediction model, dot gain } \\
\text { and ink spreading according to print order }\end{array}$ & 3.79 & 1.57 & 0 & 4.70 & 1.95 & 14 \\
\hline $\begin{array}{l}\text { New spectral prediction model, dot gain } \\
\text { and ink spreading for all superposition } \\
\text { conditions }\end{array}$ & 3.92 & 1.35 & 0 & 4.56 & 1.60 & 8 \\
\hline
\end{tabular}

$\left(1-r_{s}\right) r_{g}\left(1-r_{i}\right)\left(1-a+a t^{2}\right)$

at the second exit, the attenuation is

$\left(1-r_{s}\right) r_{g}\left(1-r_{i}\right)\left(r_{g} r_{i}\right)\left(1-a+a t^{2} t^{2}\right)$

at the third exit, the attenuation is

$$
\left(1-r_{s}\right) r_{g}\left(1-r_{i}\right)\left(r_{g} r_{i}\right)^{2}\left(1-a+a t^{2} t^{4}\right),
$$

and at the $n$th exit, the attenuation is

$$
\left(1-r_{s}\right) r_{g}\left(1-r_{i}\right)\left(r_{g}^{n-1} r_{i}^{n-1}\right)\left[(1-a)+a t^{2} t^{2(n-1)}\right] .
$$

The sum of all light components that exit after an infinite number of reflections yields the reflection spectrum

$R(\lambda)=\left(1-r_{s}\right)\left(1-r_{i}\right)\left[\frac{(1-a) r_{g}}{1-\left(r_{i} r_{g}\right)}+\frac{a r_{g} t^{2}}{1-\left(r_{i} r_{g} t^{2}\right)}\right]$

Equation (9) reflects the application of the Saunderson correction $^{26}$ accounting for multiple internal reflections at the paper-air interface: the first term within the sum models the paper without ink (internal reflectance $r_{g}$ ), and the second term models the paper printed with the solid ink (internal reflectance: $r_{g} t^{2}$ ). For single ink halftones, Eq. (9) can also be conceived as a Saunderson corrected MurrayDavis equation.

The enhanced model for three inks [Eq. (10)] comprises a part $b$ of light propagated along short and middle distances [Eq. (9), generalized to eight colorants] and a part $(1-b)$ of the light propagated along long distances [Eq. (6)].

$$
\begin{aligned}
R(\lambda)= & K r_{s}+\left(1-r_{s}\right) r_{g}\left(1-r_{i}\right)\left[b \sum_{j=1}^{8} \frac{a_{j} t_{j}^{2}}{1-r_{i} r_{g} t_{j}^{2}}\right. \\
& \left.+(1-b) \frac{\left(\sum_{j=1}^{8} a_{j} t_{j}\right)^{2}}{1-r_{g} r_{i} \sum_{j=1}^{8} a_{j} t_{j}^{2}}\right] .
\end{aligned}
$$

In Eq. (10), the part weighted by factor $b$ represents the Saunderson-corrected Neugebauer component (MurrayDavis equation extended to multiple colorants) and the part weighted by factor $(1-b)$ represents the Clapper-Yule component.

To obtain factor $b$ for a given screen frequency, we establish the prediction accuracy for 729 patches and select the value for $b$ that yields the smallest mean $\Delta E_{a b}^{*}$ error between predicted and measured reflection spectra. For cyan, magenta, and yellow offset printing on matte coated paper, with screens mutually rotated by $30 \mathrm{deg}$ and a screen frequency of $75 \mathrm{lpi}$, the fraction $b$ yielding the smallest mean error for all considered test patches is $b=0.6$. Under the same conditions, at $150 \mathrm{lpi}$, we obtain a smallest mean error at $b=0.1$, i.e., the enhanced spectral prediction model is very close to the classical Clapper-Yule model.

\section{Physical Dot Coverages Accounting for Ink Spreading}

In offset printing, trapping, i.e., the decrease in thickness of ink layers when two or more inks are printed one on top of another is generally considered to be a problem (Ref. 32, pp. 103-105). Our model automatically takes care of trapping by computing the internal transmittances of the red, green, blue, and black colorants from spectral reflection measurements according to Eq. (8). However, we observed an ink-spreading phenomenon when a second ink halftone 


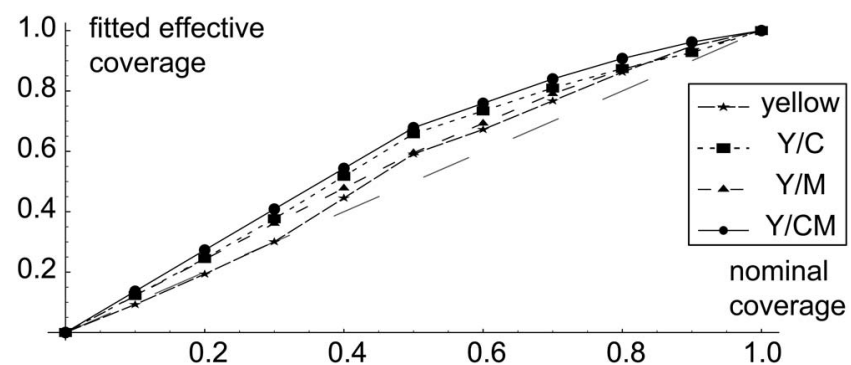

Fig. 3 Tone reproduction curves for yellow alone, yellow over solid cyan, yellow over solid magenta, and yellow over solid cyan and magenta, with effective coverages fitted according to the spectral prediction model.

is printed in superposition with a first solid ink or when a third ink halftone is printed in superposition with two solid inks. In a manner similar to the physical dot gain of a printed single ink halftone patch, ink spreading tends to enlarge the effective surface of a printed dot (Fig. 3) and tends to lower the resulting reflection spectrum, i.e., it yields slightly darker colors. However, effective surface coverages due to ink spreading are not always larger than the corresponding effective surface coverages of the same halftone patch printed alone on paper.

In the past attempts were made to model effective surface coverages when printing overlapped ink halftones. Iino and Berns introduced an empirical dot-gain scaling factor when an ink halftone is printed in superposition with another ink. ${ }^{6}$ They assume that for superpositions of more than two inks, dot-gain scaling factors are multiplicative. More recently, an ink-spreading model was developed that relies on the geometry of configurations of hexagonal dot overlaps. ${ }^{33}$ These ink-spreading models do not, however, seem to be general enough to be applicable to a large variety of printing technologies.

We develop a first model for computing effective surface coverages (physical coverages) that accounts for ink spreading. This first model assumes that when printing two successive ink layers one on top of another, ink spreading occurs only on the top layer. The top layer does not influence the effective surface coverage of the ink layer beneath it.

Let us consider a printing process where offset inks are printed by first depositing cyan, then magenta, and then yellow. We therefore fit the respective ink spreaded coverages of magenta over solid cyan $f_{c m}(m)$, yellow over solid cyan $f_{c y}(y)$, yellow over solid magenta $f_{m y}(y)$, and yellow over solid cyan and magenta $f_{c m y}(y)$ by minimizing the sum of square differences between measured spectra and spectra predicted according to Eq. (10), for a number of nominal surface coverages, e.g., 25, 50, and $75 \%$.

We also fit the effective coverages of each single ink printed on paper at the selected nominal coverages. The continuous functions $f_{c}(c), f_{m}(m), f_{y}(y), f_{c m}(m), f_{c y}(c)$, $f_{m y}(y)$, and $f_{c m y}(y)$ mapping nominal to effective coverages are obtained by linear interpolation between points in the graph (Fig. 3) formed with pairs of nominal and effective (fitted) coverage values.

In front of our new spectral prediction model, we introduce a stage taking into account effective surface coverages

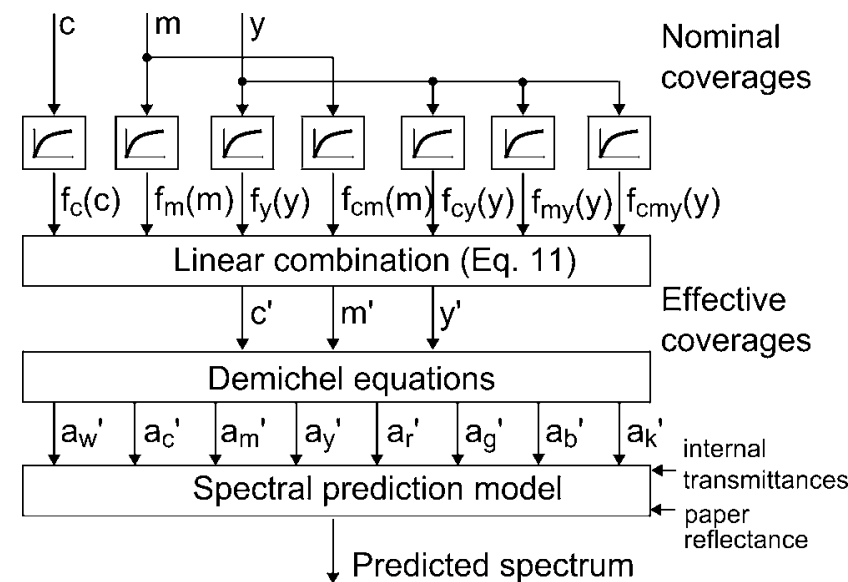

Fig. 4 Spectral prediction model with dot gain and ink spreading when an ink is printed on top of another ink.

induced by dot gain and ink spreading (Fig. 4). An input color with nominal coverages cmy is converted to an intermediate color with effective coverages $c^{\prime} m^{\prime} y^{\prime}$ obtained as a weighted sum of dot gain and ink spreading contributions.

The weighting coefficients are given by the respective effective colorant coverages.

$c^{\prime}=f_{c}(c)$ effective surface coverage of cyan only,

$m^{\prime}=f_{m}(m)\left(1-c^{\prime}\right)+f_{c m}(m) c^{\prime}$

magenta alone and magenta on top

of solid cyan (blue),

$y^{\prime}=f_{y}(y)\left(1-c^{\prime}\right)\left(1-m^{\prime}\right) \quad$ yellow alone,

$+f_{m y}(y)\left(1-c^{\prime}\right) m^{\prime}+f_{c y}(y) c^{\prime}\left(1-m^{\prime}\right)+f_{c m y}(y) c^{\prime} m^{\prime}$

yellow on top of solid magenta (red),

of solid cyan (green) and of solid cyan

\& magenta (black).

The performance of this simple ink-spreading model is illustrated by the prediction accuracies in the second row ("dot gain and ink spreading according to the print order") of the tables shown in Appendix A, both for the ClapperYule model and for our new spectral prediction model. The increase in prediction accuracy, compared with classical single ink dot gain optimization is important: for offset prints, the mean error between predicted and measured spectra, expressed in CIELAB (1976) $\Delta E_{a b}^{*}$ is reduced by a factor of 1.3 to 2 . Such an increase in prediction accuracy is true both for the Clapper-Yule model and for the new spectral prediction model.

\section{Effective Coverages in Ink Layer Superpositions: Advanced Model}

A more advanced model for computing the effective surface coverages in layer superpositions relies on the assumption that when a halftone layer is printed either beneath or on top of a solid layer, its effective surface coverage is modified. 

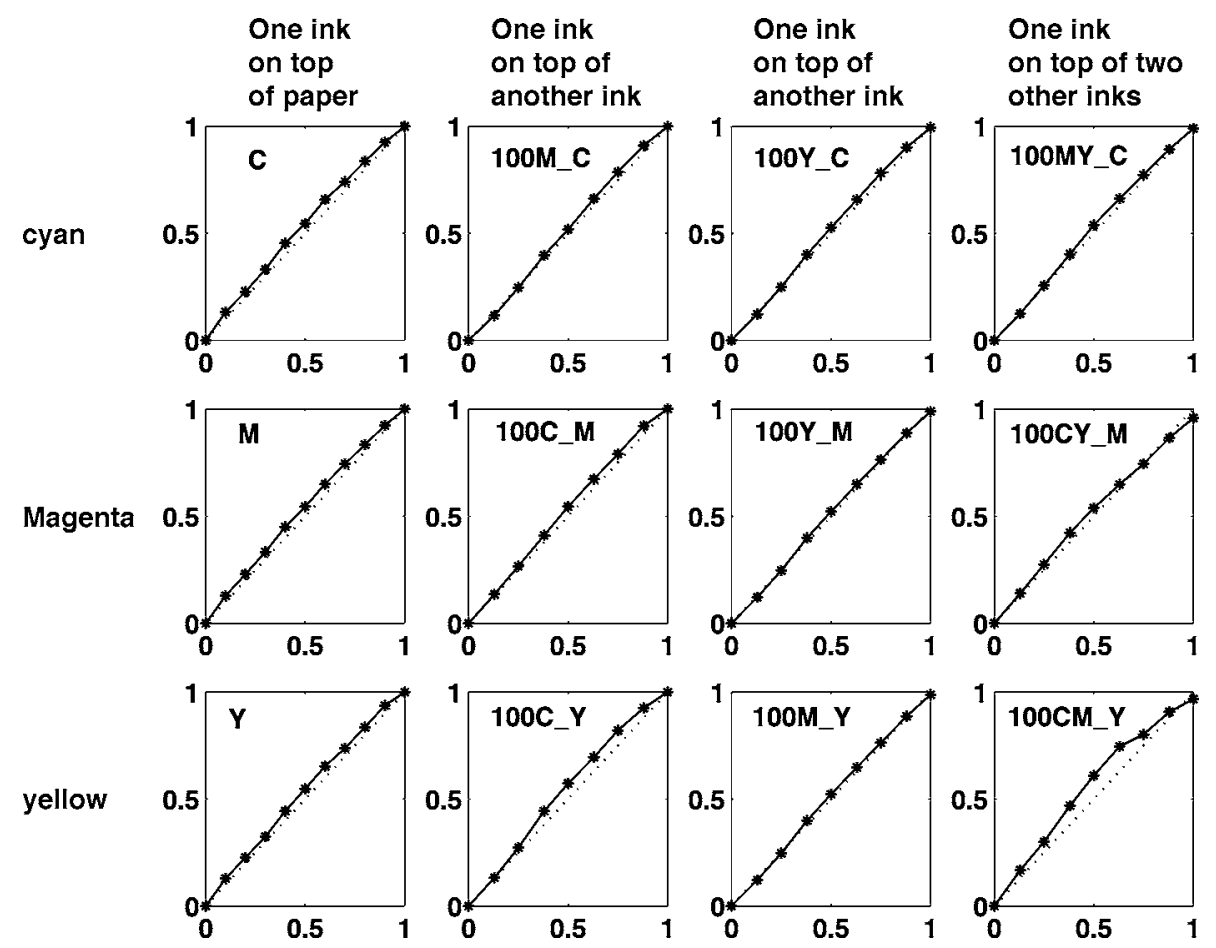

Fig. 5 Example of effective ink coverages for different superposition conditions (offset prints). Vertical axis: effective coverage. Horizontal axis: nominal coverage.

We separately model as functions of nominal coverages (1) the surface coverages of single ink halftones, (2) the surface coverages of single ink halftones superposed with one solid ink, and (3) the surface coverages of single-ink halftones superposed with two solid inks. To obtain the effective coverages $\left(c^{\prime}, m^{\prime}, y^{\prime}\right)$ of the inks of a color halftone patch as a function of the nominal coverages $(c, m, y)$, we appropriately weight the contributions of the different surface coverage functions.

During calibration of the model, the functions describing effective surface coverages of single-ink halftones printed in superposition with paper white, one solid ink or two solid inks are obtained by fitting effective surface coverages (e.g., at 25, 50, and 75\% nominal coverages) of an ink using the spectral prediction model given by Eq. (10). This enables us to associate effective (fitted) surface coverages to the nominal surface coverages, for a limited set of halftone patches of each ink, in each ink superposition condition. By linear interpolation between the so-obtained effective coverages, we obtain the functions mapping nominal to effective surface coverages of each ink for each ink superposition condition.

Let us consider three inks $i_{1}, i_{2}$, and $i_{3}$ with nominal coverages $c_{1}, c_{2}$, and $c_{3}$. The "dot gain" functions mapping nominal coverages to effective coverages for single ink halftones are $f_{1}\left(c_{1}\right), f_{2}\left(c_{2}\right)$, and $f_{3}\left(c_{3}\right)$. The "ink-spreading" functions mapping nominal coverages of an ink to effective coverages of that ink, for single-ink halftones superposed with a second solid ink and for single ink halftones superposed with two solid inks are:

for ink $i_{1}$ of coverage $c_{1}$ superposed with solid ink

$i_{2}: f_{21}\left(c_{1}\right)$, for ink $i_{1}$ of coverage $c_{1}$ superposed with solid ink $i_{3}: f_{31}\left(c_{1}\right)$,

for ink $i_{2}$ of coverage $c_{2}$ superposed with solid ink $i_{1}: f_{12}\left(c_{2}\right)$,

for ink $i_{2}$ of coverage $c_{2}$ superposed with solid ink $i_{3}: f_{32}\left(c_{2}\right)$,

for ink $i_{3}$ of coverage $c_{3}$ superposed with solid ink $i_{1}: f_{13}\left(c_{3}\right)$,

for ink $i_{3}$ of coverage $c_{3}$ superposed with solid ink $i_{2}: f_{23}\left(c_{3}\right)$,

for ink $i_{1}$ of coverage $c_{1}$ superposed with solid inks $i_{2}$ and $i_{3}: f_{231}\left(c_{1}\right)$,

for ink $i_{2}$ of coverage $c_{2}$ superposed with solid inks $i_{1}$ and $i_{3}: f_{132}\left(c_{2}\right)$,

for ink $i_{3}$ of coverage $c_{3}$ superposed with solidinks

$i_{1}$ and $i_{2}: \quad f_{123}\left(c_{3}\right)$.

In the case of three inks, these 12 functions are obtained by fitting 36 patches, i.e., three patches $(25,50$, and $75 \%$ nominal coverages) per function.

Figure 5 gives an example of effective surface coverages (round black dots at nominal coverages of 10, 20,.., 90\%) fitted according to the disclosed spectral prediction model, for wedges printed alone (left column), for wedges printed in superposition with one solid ink (second and third columns), and for wedges printed in superposition with two solid inks (right column). Wedges of cyan are shown in the 
first row, wedges of magenta in the second row, and wedges of yellow in the third row. One can see, for example, that the effective surface coverages of magenta (second row) depend if magenta is printed alone (second row, first column), in superposition with cyan (second row, second column), in superposition with yellow (second row, third column), or in superposition with cyan and magenta (second row, fourth column). One can observe the same phenomenon for the yellow wedges (third row).

Once surface coverages are fitted according to the spectral prediction model, linearly interpolating between these surface coverages (in Fig. 5, solid line segments connecting the round black dots) yields the functions mapping nominal to effective (i.e., physical) surface coverages for ink halftones printed in different superposition conditions.

To obtain the effective coverages $c_{1}^{\prime}, c_{2}^{\prime}$, and $c_{3}^{\prime}$ of a color halftone patch, it is necessary, for each ink $i_{k}$, to weight the contributions of the corresponding mapping functions $f_{k}$, $f_{l k}, f_{m k}$, and $f_{l m k}$. The weighting functions depend on the effective coverages of the considered ink alone, of the considered ink in superposition with a second ink and of the considered ink in superposition with the two other inks. For the considered system of three inks $i_{1}, i_{2}$, and $i_{3}$ with nominal coverages $c_{1}, c_{2}$, and $c_{3}$ and effective coverages $c_{1}^{\prime}, c_{2}^{\prime}$, and $c_{3}^{\prime}$, assuming that inks are printed independently of each other, by computing the relative weight, i.e., the relative surface of each superposition condition, we obtain the system of Eqs. (12). In analogy with Demichel's Eqs. (2), the proportion (relative effective surface) of a halftone patch printed with ink $i_{1}$ of coverage $c_{1}$ on paper white is $\left(1-c_{2}^{\prime}\right)\left(1-c_{3}^{\prime}\right)$. The proportion of the same patch printed on top of solid ink $i_{2}$ is $c_{2}^{\prime}\left(1-c_{3}^{\prime}\right)$, the proportion of the same patch printed on top of solid ink $i_{3}$ is $\left(1-c_{2}^{\prime}\right) c_{3}^{\prime}$ and the proportion of the same patch printed on top of solid inks $i_{2}$ and $i_{3}$ is $c_{2}^{\prime} \cdot c_{3}^{\prime}$. We obtain the following system of equations:

$$
\begin{aligned}
c_{1}^{\prime}= & f_{1}\left(c_{1}\right)\left(1-c_{2}^{\prime}\right)\left(1-c_{3}^{\prime}\right)+f_{21}\left(c_{1}\right) c_{2}^{\prime}\left(1-c_{3}^{\prime}\right) \\
& +f_{31}\left(c_{1}\right)\left(1-c_{2}^{\prime}\right) c_{3}^{\prime}+f_{231}\left(c_{1}\right) c_{2}^{\prime} c_{3}^{\prime}, \\
c_{2}^{\prime}= & f_{2}\left(c_{2}\right)\left(1-c_{1}^{\prime}\right)\left(1-c_{3}^{\prime}\right)+f_{12}\left(c_{2}\right) c_{1}^{\prime}\left(1-c_{3}^{\prime}\right) \\
& +f_{32}\left(c_{2}\right)\left(1-c_{1}^{\prime}\right) c_{3}^{\prime}+f_{132}\left(c_{2}\right) c_{1}^{\prime} c_{3}^{\prime}, \\
c_{3}^{\prime}= & f_{3}\left(c_{3}\right)\left(1-c_{1}^{\prime}\right)\left(1-c_{2}^{\prime}\right)+f_{13}\left(c_{3}\right) c_{1}^{\prime}\left(1-c_{2}^{\prime}\right) \\
& +f_{23}\left(c_{3}\right)\left(1-c_{1}^{\prime}\right) c_{2}^{\prime}+f_{123}\left(c_{3}\right) c_{1}^{\prime} c_{2}^{\prime} .
\end{aligned}
$$

This system of equations can be solved iteratively: one starts by setting initial values of $c_{1}^{\prime}, c_{2}^{\prime}$, and $c_{3}^{\prime}$ equal to the respective nominal coverages $c_{1}, c_{2}$, and $c_{3}$. After one iteration, one obtains new values for $c_{1}^{\prime}, c_{2}^{\prime}$, and $c_{3}^{\prime}$. These new values are used for the next iteration. After a few iterations, typically four to five iterations, the system stabilizes and the obtained coverages $c_{1}^{\prime}, c_{2}^{\prime}$, and $c_{3}^{\prime}$ are the effective coverages. The system of Eqs. (12) enables us therefore to compute combined effective ink surface coverages (physical dot sizes) resulting from the combination of elementary ink surface coverages present in different superposition conditions. The effective colorant coverages $a_{1}^{\prime}$, $a_{2}^{\prime}, \ldots, a_{8}^{\prime}$ are obtained from the effective coverages $c_{1}^{\prime}, c_{2}^{\prime}$,

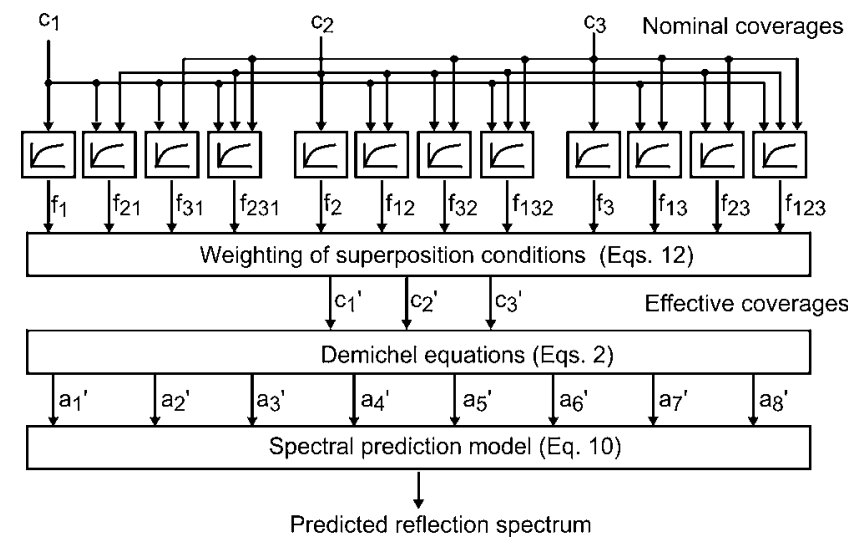

Fig. 6 Spectral prediction model with dot gain and ink spreading in all superposition conditions.

and $c_{3}^{\prime}$ of the inks according to the Demichel equations [Eqs. (2)]. The complete model comprising dot gain and ink spreading in all superposition conditions is illustrated in Fig. 6.

With the spectral reflection prediction model [Eq. (10)] and by taking into account the combined effective ink coverages (physical dot coverages), we obtain a further improvement in prediction accuracy (see Tables 1 and 2 in Appendix A). CIELAB prediction errors are reduced by another 10 to $40 \%$, compared with the simple ink spreading model (ink spreading only on top of one or two inks) described in Sec. 5.

For the same offset prints as before (Table 1 in Appendix A), thanks to the new spectral prediction and the advanced ink spreading models, at $75 \mathrm{lpi}$, with parameter $b=0.6$, a mean error between predicted reflection spectra and measured reflection spectra (in the present case 729 spectra) of $\Delta E_{a b}^{*}=1.35$ was obtained, the maximal error is $\Delta E_{a b}^{*}$ $=3.92$ and no value has a $\Delta E_{a b}^{*}$ greater than 4 . At $150 \mathrm{lpi}$, with parameter $b=0.1$, a mean error between predicted reflection spectra and measured reflection spectra (729 spectra) of $\Delta E_{a b}^{*}=1.60$ was obtained, the maximal error is $\Delta E_{a b}^{*}=4.56$ and eight values have a $\Delta E_{a b}^{*}$ greater than 4.

Although the model is presented here for the combination of a freely chosen set of three inks, it can be extended to four inks. Such an extension (Appendix C) requires to extend the Demichel equations [Eqs. (2)], the spectral prediction model [Eq. (10)], and the equations enabling us to compute the effective surface coverages according to the weights of the occurring superposition conditions [Eqs. (12)].

In contrast to Stollnitz et al. model, ${ }^{24}$ our model fits neither the transmission spectra of any of the inks nor the reflection spectrum of paper. These internal spectra are calculated from measured spectra, according to the model's equations. Our model fits only effective dot sizes when printed alone or in combination with other inks (physical dot gain, ink spreading). Nevertheless, the model accurately predicts 36 components reflection spectra. The model is therefore robust and seems to reflect, at least to a certain extent, the underlying physical phenomena. 
Hersch et al.: Spectral reflection and dot surface prediction...

Table 2 Prediction accuracy $\left(\Delta E_{a b}^{*}\right)$ for thermal transfer color halftone prints (calendered paper).

\begin{tabular}{|c|c|c|c|c|c|c|c|c|c|}
\hline $\begin{array}{l}\text { Thermal transfer } \\
\text { (ALPS MD-5000) } \\
729 \text { test samples }\end{array}$ & $\begin{array}{c}\operatorname{Max} \\
\Delta E\end{array}$ & $\begin{array}{c}\text { Mean } \\
\Delta E\end{array}$ & $\begin{array}{l}\text { Number of } \\
\text { Samples } \\
\Delta E>4\end{array}$ & $\begin{array}{c}\operatorname{Max} \\
\Delta E\end{array}$ & $\begin{array}{c}\text { Mean } \\
\Delta E\end{array}$ & $\begin{array}{c}\text { Number of } \\
\text { Samples } \\
\Delta E>4\end{array}$ & $\begin{array}{c}\operatorname{Max} \\
\Delta E\end{array}$ & $\begin{array}{l}\text { Mean } \\
\Delta E\end{array}$ & $\begin{array}{c}\text { Number of } \\
\text { Samples } \\
\Delta E>4\end{array}$ \\
\hline & \multicolumn{3}{|c|}{$50 \mathrm{Ipi}, \mathrm{b}=0.5$} & \multicolumn{3}{|c|}{75 Ipi, b=0.4 } & \multicolumn{3}{|c|}{100 Ipi, b=0.3 } \\
\hline $\begin{array}{l}\text { Clapper-Yule with } \\
\text { single ink dot gain } \\
\text { only }\end{array}$ & 7.21 & 2.96 & 170 & 7.59 & 3.34 & 241 & 8.16 & 3.30 & 224 \\
\hline $\begin{array}{l}\text { Clapper-Yule, dot gain } \\
\text { and ink spreading } \\
\text { according to } \\
\text { print order }\end{array}$ & 7.42 & 2.63 & 103 & 7.18 & 3.07 & 173 & 7.73 & 3.04 & 175 \\
\hline $\begin{array}{l}\text { Clapper-Yule, dot gain } \\
\text { and ink spreading for } \\
\text { all superposition } \\
\text { conditions }\end{array}$ & 7.74 & 2.51 & 108 & 7.06 & 2.38 & 95 & 7.73 & 2.84 & 146 \\
\hline $\begin{array}{l}\text { New spectral prediction } \\
\text { model, with single-ink } \\
\text { dot gain only }\end{array}$ & 6.63 & 2.34 & 86 & 7.73 & 3.02 & 190 & 7.33 & 3.21 & 223 \\
\hline $\begin{array}{l}\text { New spectral prediction } \\
\text { model, dot gain and ink } \\
\text { spreading according to } \\
\text { print order }\end{array}$ & 6.63 & 2.14 & 54 & 7.75 & 2.90 & 174 & 7.51 & 2.99 & 168 \\
\hline $\begin{array}{l}\text { New spectral prediction } \\
\text { model, dot gain and ink } \\
\text { spreading for all } \\
\text { superposition conditions }\end{array}$ & 4.56 & 1.50 & 5 & 5.01 & 1.51 & 13 & 6.79 & 2.50 & 78 \\
\hline
\end{tabular}

\section{Application of the Model}

The proposed spectral prediction model together with the methods of computing effective surface coverages was applied to offset and thermal transfer printing, at various screen frequencies, for the cyan, magenta, and yellow inks. Classical clustered-dot halftoning was used, with screens mutually rotated by $30 \mathrm{deg}$. We present the prediction accuracies when printing with cyan, magenta, and yellow inks on offset (Komori Lithrone 26) on coated matte paper at screen frequencies of 75 and 150 lpi and on a thermal transfer wax printer (Alps MD-5000 at 600 dpi) on calendered paper at screen frequencies of 50, 75, and 100 lpi. The tables in Appendix A give the mean prediction errors (in terms of the CIELAB $1976 \Delta E_{a b}$ values), the maximal prediction error and the number of patches having an error larger than $\Delta E_{a b}=4$. For fitting the effective dot surfaces, only 25,50 , and $75 \%$ nominal coverages were used, yielding in case of single ink dot surface optimization (dot gain) $3 \times 3=9$ patches, in case of ink spreading when printed on top of one or two inks $3 \times 7=21$ patches [Eq. (11)] and in case of ink spreading for all superposition conditions 3 $\times 12=36$ patches [Eq. (12)]. In addition, the reflectances of the paper white and of all solid ink and solid ink combinations are measured (eight patches). The model is tested on 729 patches, comprising all nominal coverage combinations at $0,13,25,38,50,63,75,88$, and $100 \%$.

The prediction results clearly show that the new spectral prediction model as well as the two ink-spreading models improve the prediction performance. One can also clearly see that combined with the new spectral prediction model, the two ink-spreading models bring considerable improvements in prediction accuracy. The ink-spreading models also bring an improvement to the classical Clapper-Yule model, but as shown by the results obtained with the Alps thermal transfer printer (Appendix A, Table 2, 50 and 75 lpi), the improvement is less pronounced than with the new spectral prediction model. The proposed models provide excellent predictions (mean CIELAB prediction error below $\Delta E_{a b}^{*}=1.6$ ) as long as the printed dot is stable (e.g., offset at 75 and $150 \mathrm{lpi}$, thermal transfer prints at 50 and 75 lpi). When the printed dot becomes somehow unstable (e.g., Table 2, Alps thermal transfer printer at 100 lpi), the proposed models still improve the prediction accuracy compared with Clapper-Yule, but provide a lower accuracy (mean $\Delta E_{a b}^{*}$ of 2.5 at 100 lpi for the thermal transfer Alps printer).

The results also confirm that the factor $b$ giving the relative weights of respectively the Saunderson-corrected Neugebauer and of the Clapper-Yule components of the spectral prediction model is related to the screen frequency. At a higher screen frequency, the ratio of lateral light scattering to screen element period is higher and therefore, the weight of the Clapper-Yule component within the new spectral prediction model becomes larger. Therefore, factor $b$, which is 
proportional to the weight of the Saunderson-corrected Neugebauer component, becomes smaller (Table 2, second line).

\section{Conclusions}

We proposed a new spectral reflection prediction model that represents a considerable progress compared with the classical Clapper-Yule model by taking into account the fact that proportionally more incident light through a given colorant surface is reflected back onto the same colorant surface than onto other colorant surfaces. The new model comprises a Saunderson-corrected Neugebauer component weighted according to a factor $b$, which specifies the part of the incident light that emerges through the same colorant as the colorant from which it entered, i.e., lateral light propagation is short compared with the screen element period. The model also comprises a Clapper-Yule component weighted according to factor $(1-b)$, which specifies the part of the incident light that emerges from all colorants, with a probability to emerge from a given colorant equal to that colorant surface coverage (lateral light propagation is middle to large, compared with the screen element period). The new spectral prediction model is therefore a weighted mean between Clapper-Yule and Saunderson-corrected Neugebauer model components.

We also proposed two models for taking into account ink spreading, a phenomenon that occurs when printing an ink halftone in superposition with one or several solid inks. Besides the physical dot gain present within a single-ink halftone print, we considered in the first model the ink spreading that occurs when an ink halftone is printed on top of one or two solid inks. In the second more advanced model, we generalized this concept to ink halftones printed on top or below solid inks. We formulated for both models systems of equations that enable us to compute effective ink coverages as a weighted mean of the individual ink coverages that occur in the different superposition conditions.

For model calibration, i.e., the establishment of the functions mapping nominal to effective surface coverages in the different superposition conditions, effective coverage values are fitted by minimizing the sum of square differences between measured and predicted reflection spectra components. In the case of three inks (cyan, magenta, and yellow), for the advanced ink-spreading model, the calibration set can be as small as 44 samples. It comprises the paper white, seven solid ink samples, and 36 halftone samples, yielding 36 fitted surface coverages for the 12 linearly interpolating functions mapping nominal to effective surface coverages.

Both the new spectral prediction model and the new methods of estimating effective coverages (dot sizes) considerably improved the predictions. Tests were carried out with 729 color patches covering the complete gamut of the output device. For offset prints, at 150 lpi (see Table 1, Appendix A), the new spectral prediction model and the advanced method for computing effective coverages improve the standard Clapper-Yule predictions by reducing the mean CIELAB (1976) error between predicted and measured spectra by a factor of 2.4 (from $\Delta E_{a b}^{*}=3.95$ to $\left.\Delta E_{a b}^{*}=1.60\right)$. At 75 lpi, the Clapper-Yule mean prediction error is reduced by a factor of 3.3 (from $\Delta E_{a b}^{*}=4.49$ to $\left.\Delta E_{a b}^{*}=1.35\right)$. Errors below to $\Delta E_{a b}^{*}=1.5$ cannot be reduced further, since they correspond to the colorimetric variations that occur when printing identical patches at different locations of the same printed page.

Since our new model fits surface coverages only when inks are printed alone or in superposition with other inks, but predicts spectra (36 components), it seems to reflect, at least to a certain extent, the underlying physical phenomena. However, further efforts are necessary to verify that indeed the computed effective surface coverages correspond to physical dot areas.

Currently, the spectral reflection prediction model together with the advanced ink-spreading model yield excellent results for clustered dots, in the case of offset and thermal transfer technologies. This may be due to the fact that both in offset and in thermal transfer technologies, ink densities remains relatively constant at each superposition condition. Further research is necessary to obtain similar results for dispersed-dot or error-diffusion halftoning algorithms, as well as for ink-jet and electrophotographic printers.

\section{Appendix A: Prediction Accuracies}

Tables 1 and 2 present prediction accuracies for offset and thermal transfer printing.

\section{Appendix B: Equivalence between Reflectance Factors Measured with an Integrated Sphere and According to a $45 \mathrm{deg} / 0 \mathrm{deg}$ Geometry}

The spectral reflectance factor of a halftone print can be obtained by dividing the spectral reflectance of the halftone print by the spectral reflectance of a perfectly white diffuser. Since for an integrated sphere, the spectral reflectance of the perfectly white diffuser is 1 at each wavelength, the reflectance factor is identical to the reflectance given by Eq. (5) in the case of a single-ink halftone.

In the case of a $45 \mathrm{deg} / 0 \mathrm{deg}$ measuring geometry, when discarding the specular component, the exact expression for the Clapper-Yule reflectance factor, measured in reference to a perfect white diffuser, is ${ }^{27}$

$$
\begin{aligned}
R^{45 \operatorname{deg} / 0 \operatorname{deg}}(\lambda)= & \left(1-r_{s}^{45 \mathrm{deg}}\right) \frac{\left(1-r_{s}^{0 \mathrm{deg}}\right)}{n_{2}^{2}} \\
& \times \frac{r_{g}(1-a+a t)^{2}}{1-r_{g} r_{i}\left(1-a+a t^{2}\right)},
\end{aligned}
$$

where $r_{s}^{45 \mathrm{deg}}$ and, respectively, $r_{s}^{0 \mathrm{deg}}$ are the Fresnel reflection factors for 45 and, respectively, 0 deg incident light at the air-coating interface and where $n_{2}$ is the index of refraction of coated paper. The symbolic Eq. (13) for the reflection factor in case of a $45 \mathrm{deg} / 0 \mathrm{deg}$ measuring geometry differs from the symbolic Eq. (5) of the reflectance in case of an integrated sphere measuring geometry only by (1 $\left.-r_{s}^{0 \mathrm{deg}}\right) / n_{2}^{2}$ being replaced with $\left(1-r_{i}\right)$. By inserting the corresponding numerical values

$\frac{\left(1-r_{s}^{0 \mathrm{deg}}\right)}{n_{2}^{2}}=\frac{(1-0.044)}{1.53^{2}}=0.408$,

and, respectively, 
$\left(1-r_{i}\right)=(1-0.614)=0.386$,

in their corresponding Eq. (13) and, respectively, Eq. (5), and by computing accordingly the paper's internal reflectance $r_{g}$ [Eq. (7)] and the ink transmittance $t_{j}$ [Eq. (8)], one can verify that for all surface coverages and ink transmittance levels, the reflection factor components computed according to Eq. (13) and according to Eq. (5) vary numerically by less than $2.5 \%$.

In terms of CIELAB (1976) $\Delta E_{a b}^{*}$ values, the distance between reflection spectra measured according to an integrated sphere and according to a $45 \mathrm{deg} / 0 \mathrm{deg}$ geometry yields color differences smaller than $\Delta E_{a b}^{*}=0.5$. As suggested by Clapper and Yule, ${ }^{21}$ we can therefore use a photospectrometer with a $45 \mathrm{deg} / 0 \mathrm{deg}$ geometry instead of an integrating sphere for carrying out the reflectance measurements necessary to obtain correct Clapper-Yule reflectance predictions. Similar considerations also apply to the Saunderson-modified Neugebauer model component and therefore to the new spectral prediction model.

\section{Appendix C: Extension of the Model to Four Inks}

The extension of the Demichel Eqs. (2) to four inks $i_{1}, i_{2}$, $i_{3}$, and $i_{4}$ with respective coverages $c_{1}, c_{2}, c_{3}$, and $c_{4}$ yields the colorant coverages:

$$
\begin{aligned}
& i_{1} \text { alone: } \quad a_{1}=c_{1}\left(1-c_{2}\right)\left(1-c_{3}\right)\left(1-c_{4}\right), \\
& i_{2} \text { alone: } \quad a_{2}=\left(1-c_{1}\right) c_{2}\left(1-c_{3}\right)\left(1-c_{4}\right), \\
& i_{3} \text { alone: } \quad a_{3}=\left(1-c_{1}\right)\left(1-c_{2}\right) c_{3}\left(1-c_{4}\right), \\
& i_{4} \text { alone: } \quad a_{4}=\left(1-c_{1}\right)\left(1-c_{2}\right)\left(1-c_{3}\right) c_{4}, \\
& i_{1} \text { and } i_{2}: \quad a_{5}=c_{1} c_{2}\left(1-c_{3}\right)\left(1-c_{4}\right), \\
& i_{1} \text { and } i_{3}: \quad a_{6}=c_{1}\left(1-c_{2}\right) c_{3}\left(1-c_{4}\right), \\
& i_{1} \text { and } i_{4}: \quad a_{7}=c_{1}\left(1-c_{2}\right)\left(1-c_{3}\right) c_{4}, \\
& i_{2} \text { and } i_{3}: \quad a_{8}=\left(1-c_{1}\right) c_{2} c_{3}\left(1-c_{4}\right), \\
& i_{2} \text { and } i_{4}: \quad a_{9}=\left(1-c_{1}\right) c_{2}\left(1-c_{3}\right) c_{4}, \\
& i_{3} \text { and } i_{4}: \quad a_{10}=\left(1-c_{1}\right)\left(1-c_{2}\right) c_{3} c_{4}, \\
& i_{1}, i_{2}, \text { and } i_{3}: \quad a_{11}=c_{1} c_{2} c_{3}\left(1-c_{4}\right), \\
& i_{2}, i_{3}, \text { and } i_{4}: \quad a_{12}=\left(1-c_{1}\right) c_{2} c_{3} c_{4}, \\
& i_{1}, i_{3}, \text { and } i_{4}: \quad a_{13}=c_{1}\left(1-c_{2}\right) c_{3} c_{4}, \\
& i_{1}, i_{2}, \text { and } i_{4}: \quad a_{14}=\left(1-c_{1}\right)\left(1-c_{2}\right) c_{3}\left(1-c_{4}\right), \\
& i_{1}, i_{2}, i_{3}, \text { and } i_{4}: \quad a_{15}=c_{1} c_{2} c_{3} c_{4}, \\
& \text { white: } \quad a_{16}=\left(1-c_{1}\right)\left(1-c_{2}\right)\left(1-c_{3}\right)\left(1-c_{4}\right) .
\end{aligned}
$$

The extension of Eq. (10) to four inks leads to

$$
\begin{aligned}
R(\lambda)= & K r_{s}+\left(1-r_{s}\right) r_{g}\left(1-r_{i}\right)\left[b \sum_{j=1}^{16} \frac{a_{j} t_{j}^{2}}{1-r_{i} r_{g} t_{j}^{2}}\right. \\
& \left.+(1-b) \frac{\left(\sum_{j=1}^{16} a_{j} t_{j}\right)^{2}}{1-r_{g} r_{i} \sum_{j=1}^{16} a_{j} t_{j}^{2}}\right] .
\end{aligned}
$$

Finally, the extension of Eq. (12) to four inks leads to

$$
\begin{aligned}
c_{1}^{\prime}= & f_{1}\left(c_{1}\right)\left(1-c_{2}^{\prime}\right)\left(1-c_{3}^{\prime}\right)\left(1-c_{4}^{\prime}\right)+f_{41}\left(c_{1}\right)\left(1-c_{2}^{\prime}\right)(1 \\
& \left.-c_{3}^{\prime}\right) c_{4}^{\prime}+f_{21}\left(c_{1}\right) c_{2}^{\prime}\left(1-c_{3}^{\prime}\right)\left(1-c_{4}^{\prime}\right)+f_{241}\left(c_{1}\right) c_{2}^{\prime}(1 \\
& \left.-c_{3}^{\prime}\right) c_{4}^{\prime}+f_{31}\left(c_{1}\right)\left(1-c_{2}^{\prime}\right) c_{3}^{\prime}\left(1-c_{4}^{\prime}\right)+f_{341}\left(c_{1}\right)(1
\end{aligned}
$$

$$
\left.-c_{2}^{\prime}\right) c_{3}^{\prime} c_{4}^{\prime}+f_{231}\left(c_{1}\right) c_{2}^{\prime} c_{3}^{\prime}\left(1-c_{4}^{\prime}\right)+f_{2341}\left(c_{1}\right) c_{2}^{\prime} c_{3}^{\prime} c_{4}^{\prime},
$$

$$
\begin{aligned}
c_{2}^{\prime}= & f_{2}\left(c_{2}\right)\left(1-c_{1}^{\prime}\right)\left(1-c_{3}^{\prime}\right)\left(1-c_{4}^{\prime}\right)+f_{42}\left(c_{2}\right)\left(1-c_{1}^{\prime}\right)(1 \\
& \left.-c_{3}^{\prime}\right) c_{4}^{\prime}+f_{12}\left(c_{2}\right) c_{1}^{\prime}\left(1-c_{3}^{\prime}\right)\left(1-c_{4}^{\prime}\right)+f_{142}\left(c_{2}\right) c_{1}^{\prime}(1 \\
& \left.-c_{3}^{\prime}\right) c_{4}^{\prime}+f_{32}\left(c_{2}\right)\left(1-c_{1}^{\prime}\right) c_{3}^{\prime}\left(1-c_{4}^{\prime}\right)+f_{342}\left(c_{2}\right)(1 \\
& \left.-c_{1}^{\prime}\right) c_{3}^{\prime} c_{4}^{\prime}+f_{132}\left(c_{2}\right) c_{1}^{\prime} c_{3}^{\prime}\left(1-c_{4}^{\prime}\right)+f_{1342}\left(c_{2}\right) c_{1}^{\prime} c_{3}^{\prime} c_{4}^{\prime},
\end{aligned}
$$

$$
\begin{aligned}
c_{3}^{\prime}= & f_{3}\left(c_{3}\right)\left(1-c_{1}^{\prime}\right)\left(1-c_{2}^{\prime}\right)\left(1-c_{4}^{\prime}\right)+f_{43}\left(c_{3}\right)\left(1-c_{1}^{\prime}\right)(1 \\
& \left.-c_{2}^{\prime}\right) c_{4}^{\prime}+f_{13}\left(c_{3}\right) c_{1}^{\prime}\left(1-c_{2}^{\prime}\right)\left(1-c_{4}^{\prime}\right)+f_{143}\left(c_{3}\right) c_{1}^{\prime}(1 \\
& \left.-c_{2}^{\prime}\right) c_{4}^{\prime}+f_{23}\left(c_{3}\right)\left(1-c_{1}^{\prime}\right) c_{2}^{\prime}\left(1-c_{4}^{\prime}\right)+f_{243}\left(c_{3}\right)(1 \\
& \left.-c_{1}^{\prime}\right) c_{2}^{\prime} c_{4}^{\prime}+f_{123}\left(c_{3}\right) c_{1}^{\prime} c_{2}^{\prime}\left(1-c_{4}^{\prime}\right)+f_{1243}\left(c_{3}\right) c_{1}^{\prime} c_{2}^{\prime} c_{4}^{\prime},
\end{aligned}
$$

$$
\begin{aligned}
c_{4}^{\prime}= & f_{4}\left(c_{4}\right)\left(1-c_{1}^{\prime}\right)\left(1-c_{2}^{\prime}\right)\left(1-c_{3}^{\prime}\right)+f_{14}\left(c_{4}\right) c_{1}^{\prime}\left(1-c_{2}^{\prime}\right)(1 \\
& \left.-c_{3}^{\prime}\right)+f_{24}\left(c_{4}\right)\left(1-c_{1}^{\prime}\right) c_{2}^{\prime}\left(1-c_{3}^{\prime}\right)+f_{34}\left(c_{4}\right)\left(1-c_{1}^{\prime}\right)(1 \\
& \left.-c_{2}^{\prime}\right) c_{3}^{\prime}+f_{124}\left(c_{4}\right) c_{1}^{\prime} c_{2}^{\prime}\left(1-c_{3}^{\prime}\right)+f_{134}\left(c_{4}\right) c_{1}^{\prime}\left(1-c_{2}^{\prime}\right) c_{3}^{\prime} \\
& +f_{234}\left(c_{4}\right)\left(1-c_{1}^{\prime}\right) c_{2}^{\prime} c_{3}^{\prime}+f_{1234}\left(c_{4}\right) c_{1}^{\prime} c_{2}^{\prime} c_{3}^{\prime} .
\end{aligned}
$$

Equations (16)-(18) completely define the new model for the case of four inks.

\section{Acknowledgments}

We thank Mathieu Hebert for fruitful discussions as well as the Swiss National Science Foundation for its financial support (grants 2000-065167 and 200020-105119/1).

\section{References}

1. D. R. Wyble and R. S. Berns, "A critical review of spectral models applied to binary color printing," Color Res. Appl. 25(1), 4-19 (2000).

2. R. Balasubramanian, "Optimization of the spectral Neugebauer model for printer characterization," J. Electron. Imaging 8(2), 156166 (1999).

3. J. A. C. Yule and W. J. Nielsen, "The penetration of light into paper and its effect on halftone reproductions," Proc. Annu. Tech. Meet. Tech. Assoc. Graphic Arts 3, 65-76 (1951).

4. J. A. S. Viggiano, "Modeling the color of multi-colored halftones," Proc.-TAGA, 44-62 (1990).

5. K. Iino and R. S. Berns, "Building color management modules using linear optimization I. Desktop,” J. Imaging Sci. Technol. 42(1), 79-94 (1998).

6. K. Iino and R. S. Berns, "Building color management modules using linear optimization II. Prepress system for offset printing," J. Imaging Sci. Technol. 42(2), 99-114 (1998).

7. H. R. Kang, Color Technology for Electronic Imaging Devices, SPIE Optical Engineering Press, Bellingham, WA (1997).

8. H. E. J. Neugebauer, "Die theoretischen Grundlagen des Mehrfarbendrucks," Z. Wissen. Photog. 36, 36-73 (1937); reprinted in Neugebauer Semin. Color Reprod., Proc. SPIE 1184, 194-202 (1989).

9. M. E. Demichel, Procédé 26, 17-21 (1924).

10. A. Murray, "Monochrome reproduction in photoengraving," J. Franklin Inst. 221, 721-724 (1936).

11. H. R. Kang, "Applications of color mixing models to electronic printing," J. Electron. Imaging 3(3), 276-287 (1994).

12. F. R. Ruckdeschel and O. G. Hauser, "Yule-Nielsen effect in printing: a physical analysis," Appl. Opt. 17(21), 3376-3383 (1978).

13. J. A. C. Yule, D. J. Howe, and J. H. Altman, "The effect of the spread function of paper on halftone reproduction," Tappi J. 50(7), 337-344 (1967).

14. H. Wakeshima, T. Kunishi, and S. Kaneko, "Light scattering in paper and its effect on halftone reproduction," J. Opt. Soc. Am. 58, 272-273 (1968).

15. P. G. Engeldrum and B. Pridham, "Application of turbid medium theory to paper spread function measurements," in Proc.-TAGA 47 pp. 339-352 (1995).

16. S. Gustavson, "Color gamut of halftone reproduction," J. Imaging Sci. Technol. 41(3), 283-290 (1997). 
17. J. S. Arney, "A probability description of the Yule-Nielsen effect I," J. Imaging Sci. Technol. 41(6), 633-636 (1997).

18. G. Rogers, "Optical dot gain: lateral scattering probabilities," J. Imaging Sci. Technol. 42(4), 341-345 (1998).

19. L. Yang, R. Gooran, and B. Kruse, "Simulation of optical dot gain in multichromatic tone production," J. Imaging Sci. Technol. 45(2), 198-204 (2001).

20. L. Yang, R. Lenz, and B. Kruse, "Light scattering and ink penetration effects on tone reproduction," J. Opt. Soc. Am. A 18(2), 360-366 (2001).

21. F. R. Clapper and J. A. C. Yule, "The effect of multiple internal reflections on the densities of halftone prints on paper," J. Opt. Soc. Am. 43, 600-603 (1953)

22. G. Rogers, "A generalized Clapper-Yule model of halftone reflectance," Color Res. Appl. 25(6), 402-407 (2000).

23. P. Emmel and R. D. Hersch, "A unified model for color prediction of halftoned prints," J. Imaging Sci. Technol. 44(4), 351-359 (2000).

24. E. J. Stollnitz, V. Ostromoukhov, and D. Salesin, "Reproducing color images using custom inks," in Proc. SIGGRAPH 1998, Computer Graphics Proc., Annual Conference Series, pp. 219-228, ACM Press (1998).

25. P. Kubelka, "New contributions to the optics of intensely lightscattering material, part II: non-homogeneous layers," J. Opt. Soc. Am. 44, 330-335 (1954).

26. J. L. Saunderson, "Calculation of the color pigmented plastics," $J$. Opt. Soc. Am. 32, 727-736 (1942).

27. M. Hebert and R. D. Hersch, "Classical print reflection models, a radiometric approach," J. Imaging Sci. Technol. 48(4), 363-374 (2004).

28. E. Hecht, Schaum's Outline of Optics, McGraw-Hill, New York (1974).

29. D. B. Judd, "Fresnel reflection of diffusely incident light," J. Res. Natl. Bur. Stand. 29, 329-332 (1942).

30. P. Emmel, "Physical models for color prediction," in Digital Color Imaging, G. Sharma, Ed., pp. 173-238, CRC Press, Boca Raton, FL (2003).

31. S. Inoue, N. Tsumara, and Y. Miyake, "Measuring MTF of paper by sinusoidal test pattern projection," J. Imaging Sci. Technol. 41(6), 657-661 (1997)

32. H. Kipphan, Handbook of Print Media, Springer-Verlag (2001).

33. P. Emmel and R. D. Hersch, "Modeling ink spreading for color prediction," J. Imaging Sci. Technol. 46(3), 237-246 (2002).

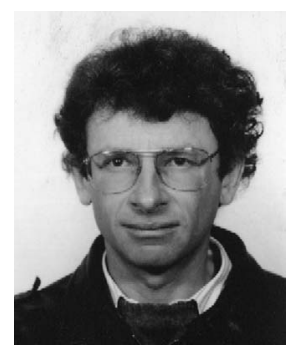

Roger D. Hersch is a professor of computer science and heads the Peripheral Systems Laboratory at the Ecole Polytechnique Fédérale de Lausanne (EPFL), Switzerland. He received his engineering and $\mathrm{PhD}$ degrees, respectively, from ETH, Zurich, in 1975 and from EPFL in 1985. He has published over 100 scientific papers, is the editor of four books, and is inventor or coinventor of many patent applications. He is interested in novel imaging techniques (color prediction, color reproduction, artistic imaging, security print- ing, anticounterfeiting) and in high-performance server applications (imaging servers, PC clusters).

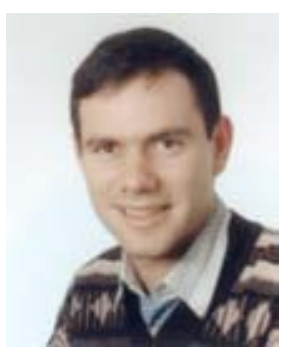

Patrick Emmel heads color communication in the Masterbatch Division of Clariant. His research interests include color prediction, mathematical modeling, color printing, and the history of mathematics. He graduated as an electronics and physics engineer from CPE Engineering School, Lyon, France in 1993, and in the same year he received an MSc degree in computer science from the University Jean Monnet of Saint Etienne, France. Before joining Clariant, he was research assistant at the Peripheral Systems Laboratory of the Ecole Polytechnique Fédérale de Lausanne (EPFL) in Lausanne, Switzerland. He received his $\mathrm{PhD}$ degree in computer science from EPFL in 1998.

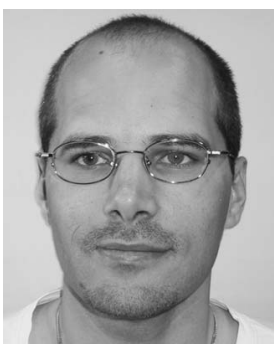

Fabien Collaud received his engineering degree from the Ecole Polytechnique Fédérale de Lausanne (EPFL), Switzerland, in 2000. From 2000 to 2004, he was an R\&D engineer with EPFL and contributed to color prediction models for offset printing.

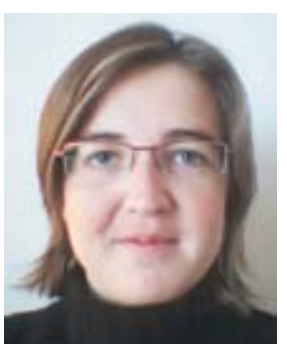

Frédérique Crété received her engineering and MS degrees in 2003, respectively, from ISTASE and from University Jean Monnet of Saint Etienne, France. She was active for 2 years at the Ecole Polytechnique Fédérale de Lausanne (EPFL) in the fields of color prediction and reproduction. She is currently working toward her PhD degree, aiming at reducing MPEG artifacts. 